Algebra univers. 52 (2004) 215-239

$0002-5240 / 04 / 030215-25$

DOI $10.1007 / \mathrm{s} 00012-004-1870-4$

(C) Birkhäuser Verlag, Basel, 2004

Algebra Universalis

\title{
Minimal varieties of residuated lattices
}

\author{
Nikolaos Galatos \\ In Celebration of the Sixtieth Birthday of Ralph N. McKenzie
}

\begin{abstract}
In this paper we investigate the atomic level in the lattice of subvarieties of residuated lattices. In particular, we give infinitely many commutative atoms and construct continuum many non-commutative, representable atoms that satisfy the idempotent law; this answers Problem 8.6 of [12]. Moreover, we show that there are only two commutative idempotent atoms and only two cancellative atoms. Finally, we study the connections with the subvariety lattice of residuated bounded-lattices. We modify the construction mentioned above to obtain a continuum of idempotent, representable minimal varieties of residuated bounded-lattices and illustrate how the existing construction provides continuum many covers of the variety generated by the three-element non-integral residuated boundedlattice.
\end{abstract}

\section{Introduction}

A residuated lattice, or residuated lattice-ordered monoid, is an algebra $\mathbf{L}=$ $\langle L, \wedge, \vee, \cdot, \backslash, /, e\rangle$ such that $\langle L, \wedge, \vee\rangle$ is a lattice, $\langle L, \cdot, e\rangle$ is a monoid and multiplication is residuated with respect to the order by the division operations $\backslash, /$; i.e., for all $a, b, c \in L$,

$$
a \cdot b \leq c \Leftrightarrow a \leq c / b \Leftrightarrow b \leq a \backslash c
$$

The last two equivalences can be written in equational form, see [5], so the class $\mathcal{R} \mathcal{L}$ of all residuated lattices is a variety.

Residuated lattices were first introduced in the late 1930's in a more restrictive form by M. Ward and R. P. Dilworth, see [18], in their attempt to generalize the ideal lattices of rings with identity.

Residuated lattices provide algebraic semantics for substructural logics, logics that, when presented in a sequent calculus system, lack some of the three structural rules of exchange, contraction and weakening, see $[12,15,16]$. On the other hand, residuated lattices generalize well-studied algebraic structures, including latticeordered groups, Brouwerian algebras and (generalized) $M V$-algebras. The structure

Presented by J. Berman.

Received August 1, 2003; accepted in final form April 27, 2004.

2000 Mathematics Subject Classification: 06F05; 08B15, 03B47, 03G10.

Key words and phrases: Residuated lattice, subvariety lattice, minimal variety, atom. 
theory of residuated lattices, considered in the generality we adopt here, was studied only recently by K. Blount and C. Tsinakis [5]. A growing literature on the subject and on related structures includes $[3,6,7,9,10,17]$; for a survey of residuated lattices and for additional references, see [12].

The goal of this paper is the study of minimal non-trivial subvarieties of $\mathcal{R} \mathcal{L}$. First we note that every finite strictly simple residuated lattice generates an atom in the lattice $\mathbf{L}(\mathcal{R L})$ of subvarieties of $\mathcal{R} \mathcal{L}$. In Section 4 , we give a countably infinite collection of commutative atoms and provide a basis of equations for each such variety. It is also shown that there are only two cancellative atoms. In [12] continuum many atoms of $\mathbf{L}(\mathcal{R} \mathcal{L})$ are produced. All of them satisfy the identity $x^{4} \approx x^{3}$, but not the identity $x^{3} \approx x^{2}$. In Section 5 , we improve this result by constructing continuum many atoms that satisfy the idempotent law $x^{2} \approx x$ and distributivity; actually all the atoms constructed are representable. Moreover, we prove that there are only two commutative idempotent atoms, a fact that indicates the necessity of non-commutativity in the previous construction.

The residuated lattices that generate the uncountably many atoms given in Section 5, all have a totally ordered lattice reduct. They also have a conservative multiplication, so the subalgebra generation uses only the two division operations. Actually, just the terms $e / x$ and $x \backslash e$ are enough. The underlying lattice is the ordinal sum of the integers, a distinct element, that plays the role of the multiplicative identity, and of the dual of the integers. The product of two elements is the farthest from the identity, namely the one with the smallest integer index, and one is given a binary choice for the product of any pair of positive and negative elements with the same index. One can encode these choices into a function from the integers to the doubleton $\{0,1\}$. A preorder can be defined on these functions, also called bi-infinite words, based on the set of their finite subwords. It turns out that the residuated lattice determined by a minimal, with respect to the preorder, equivalence class of bi-infinite words generates an atom in $\mathbf{L}(\mathcal{R} \mathcal{L})$. These minimal words have been studied under different names and it is well known that they form continuum many classes, thus yielding the desired result.

In Section 6, we describe a construction that, given a suitable pair of a residuated lattice $\mathbf{L}$ and a residuated bounded-lattice $\mathbf{K}$, produces a residuated bounded-lattice $\mathbf{K}[\mathbf{L}]$; a residuated bounded-lattice is the expansion of a bounded residuated lattice such that the bounds are included in the language. If $\mathbf{K}$ is finite and strictly simple and $\mathbf{V}(\mathbf{L})$ is an atom, then $\mathbf{V}(\mathbf{K}[\mathbf{L}])$ is a cover of $\mathbf{V}(\mathbf{K})$. We apply this result to the atoms of Section 5 to obtain a continuum of covers of the three-element non-integral residuated bounded-lattice. Finally, we modify the algebras in Section 4 to obtain continuum many idempotent, representable, residuated bounded-lattice atoms. 


\section{Preliminaries}

Note that $\mathcal{R} \mathcal{L}$ is a congruence distributive variety, since the $\{\wedge, \vee\}$-reduct of a residuated lattice is a lattice. Actually, it is also congruence permutable, see $[4,12]$, so it is arithmetical. Observe that in a residuated lattice, the division operations are determined by multiplication and the order; in particular, $x / y=\bigvee\{z \mid z y \leq x\}$ and $y \backslash x=\bigvee\{z \mid y z \leq x\}$

In a residuated lattice term, we assume that multiplication has priority over the division operations, which, in turn, have priority over the lattice operations. So, for example, we write $x / y z \wedge u \backslash v$ for $[x /(y z)] \wedge(u \backslash v)$. We will be using the inequality $t \leq s$ instead of the equalities $t=t \wedge s$ and $t \vee s=s$ in order to simplify the presentation, whenever appropriate.

Lemma 2.1. [5] Residuated lattices satisfy the following identities:

(1) $x(y \vee z) \approx x y \vee x z$ and $(y \vee z) x \approx y x \vee z x$

(2) $x \backslash(y \wedge z) \approx(x \backslash y) \wedge(x \backslash z)$ and $(y \wedge z) / x \approx(y / x) \wedge(z / x)$

(3) $x /(y \vee z) \approx(x / y) \wedge(x / z)$ and $(y \vee z) \backslash x \approx(y \backslash x) \wedge(z \backslash x)$

(4) $(x / y) y \leq x$ and $y(y \backslash x) \leq x$

(5) $x(y / z) \leq(x y) / z$ and $(z \backslash y) x \leq z \backslash(y x)$

(6) $(x / y) / z \approx x /(z y)$ and $z \backslash(y \backslash x) \approx(y z) \backslash x$

(7) $x \backslash(y / z) \approx(x \backslash y) / z$

(8) $x / e \approx x \approx e \backslash x$

(9) $e \leq x / x$ and $e \leq x \backslash x$

Moreover, if a residuated lattice has a least element $\perp$, then it has a greatest element $\top$, as well, and $\top=\perp / \perp=\perp \backslash \perp$.

It follows from (1), (2) and (3) of the previous lemma that multiplication is order preserving, and that the division operations are order preserving in the numerator and order reversing in the denominator.

A residuated lattice is called commutative (respectively, cancellative, idempotent), if its monoid reduct is commutative (respectively, cancellative, idempotent). It is called integral, if its lattice reduct has a top element and the latter coincides with the multiplicative identity $e$. It is easy to see that a residuated lattice is cancellative if and only if it satisfies the identities $x \backslash x y \approx y \approx y x / x$, see [2], hence the class of all cancellative residuated lattices forms a variety. Note that in a residuated lattice commutativity is equivalent to $x / y \approx y \backslash x$.

The negative cone of a residuated lattice $\mathbf{L}=\langle L, \wedge, \vee, \cdot, \backslash, /, e\rangle$ is the algebra $\mathbf{L}^{-}=\left\langle L^{-}, \wedge, \vee, \cdot \backslash_{\mathbf{L}^{-}}, / \mathbf{L}^{-}, e\right\rangle$, where $L^{-}=\{x \in L \mid x \leq e\}, x \backslash_{\mathbf{L}^{-}} y=x \backslash y \wedge e$ and 
$x / \mathbf{L}^{-} y=x / y \wedge e$. It is easy to verify that $\mathbf{L}^{-}$is a residuated lattice; obviously, $\mathbf{L}^{-}$ is integral.

A generalized $B L$-algebra (GBL-algebra) is a residuated lattice that satisfies the identities $((x \wedge y) / y) y \approx x \wedge y \approx y(y \backslash(x \wedge y))$. A generalized $M V$-algebra (GMValgebra $)$ is a residuated lattice that satisfies the identities $x /((x \vee y) \backslash x) \approx x \vee y \approx$ $(x /(x \vee y)) \backslash x$. For motivation on these definitions, connections to logic and further results, see $[2,10]$. It can be shown that GMV-algebras are GBL-algebras, and that GBL-algebras have a distributive lattice reduct. A Brouwerian algebra is (term equivalent to) a residuated lattice that satisfies the law $x y \approx x \wedge y$. It can be easily verified that every Brouwerian algebra is an integral GBL-algebra. We denote the corresponding varieties by $\mathcal{G B} \mathcal{L}, \mathcal{G M V}$ and $\mathcal{B} r$.

For each element $a$ in a residuated lattice $\mathbf{L}$, we define two unary polynomials $\rho_{a}(x)=a x / a \wedge e$ and $\lambda_{a}(x)=a \backslash x a \wedge e$, the right and left conjugate of $x$ by $a$. An iterated conjugate is a composition, with respect to their arguments, of a number of left and right conjugates.

A subset $N$ of $L$ is called normal, if it is closed under conjugation; i.e., for all $x$ in $N$ and $a$ in $L, \lambda_{a}(x), \rho_{a}(x) \in N$. A subset $X$ of $L$ is called convex, if for every $x, y$ in $X$ and $z$ in $L, x \leq z \leq y$ implies that $z$ is in $X$.

Theorem 2.2. [5] The convex normal subalgebras of a residuated lattice $\mathbf{L}$ form a lattice, $\mathbf{C N S}(\mathbf{L})$, which is isomorphic to the congruence lattice, $\mathbf{C o n L}$, of $\mathbf{L}$ via $S \mapsto \theta_{S}=\left\{(a, b) \in L^{2} \mid(a / b \wedge e)(b / a \wedge e) \in S\right\}$ and $\theta \mapsto[e]_{\theta}$, the $\theta$-class of $e$.

A residuated lattice is called representable if it is isomorphic to a product of totally ordered residuated lattices. We denote the variety of all representable residuated lattices by $\mathcal{R} \mathcal{L}^{C}$; the choice of the superscript is motivated by the fact that the variety is generated by residuated lattices that are chains.

Theorem 2.3. $[5,12]$ The variety $\mathcal{R} \mathcal{L}^{C}$ is axiomatized relative to $\mathcal{R} \mathcal{L}$ by the identity $\lambda_{z}(x /(x \vee y)) \vee \rho_{w}(y /(x \vee y)) \approx e$.

\section{General facts about atoms}

In this section we describe the finitely generated atoms of $\mathbf{L}(\mathcal{R L})$.

A non-trivial algebra $\mathbf{A}$ is called strictly simple, if it lacks non-trivial proper subalgebras and congruences. Recall that, by Theorem 2.2, congruences on residuated lattices correspond to convex normal subalgebras. So, the absence of non-trivial proper subalgebras in a residuated lattice is enough to establish strict simplicity. 
Proposition 3.1. Let $a$ be a non-identity element of a strictly simple, lowerbounded residuated lattice $\mathbf{A}$, and let $t$ be a unary term such that $\mathbf{A}$ satisfies $t(x)=a$, for all $x \in A, x \neq e$. Then, the variety generated by $\mathbf{A}$ is an atom in the subvariety lattice.

Proof. Let $\mathcal{V}$ be the variety generated by A. By Jónsson's Lemma, for congruence distributive varieties, the subdirectly irreducible algebras of $\mathcal{V}$ are contained in $\mathbf{H S P}_{u}(\mathbf{A})$. So, if $\mathbf{D} \in \mathcal{V}_{S I}$, there exists an ultrapower $\mathbf{B}$ of $\mathbf{A}$ and a non-trivial subalgebra $\mathbf{C}$ of $\mathbf{B}$ such that $\mathbf{D}=f(\mathbf{C})$, for some homomorphism $f$. Since $\mathbf{A}$ is strictly simple, thus generated by any of its non-identity elements, we can assume, without loss of generality, that $a=\perp$, the least element of $\mathbf{A}$. Note that $\mathbf{A}$ satisfies the first order formula:

$$
(\forall x, y, z)(x \neq e \neq y \rightarrow t(x)=t(y) \leq z),
$$

thus, so does $\mathbf{B}$, by properties of the ultrafilter construction. So, $\mathbf{B}$ has a least element $\perp^{\prime}$, which is actually contained in all non-trivial subalgebras of $\mathbf{B}$.

Since the least element is almost term definable and $\mathbf{A}$ is generated by $\perp$, the subalgebra $\mathbf{F}$ of $\mathbf{C}$ generated by $\perp^{\prime}$ is isomorphic to $\mathbf{A}$; hence $\mathbf{F}$ is strictly simple. If any two elements of $F$ have the same image under $f$, then $f(F)=\{e\}$; thus $f\left(\perp^{\prime}\right)=$ $f(e)$. Since the identity element of a residuated lattice is its least element, only if the residuated lattice is trivial, we get $f(C)=\{e\}$, a contradiction. Consequently, $f(\mathbf{F}) \subseteq \mathbf{D}$ is isomorphic to $\mathbf{F}$. Thus, $\mathbf{A}$ is isomorphic to a subalgebra of every subdirectly irreducible member of $\mathcal{V}$; hence $\mathcal{V}$ is an atom.

Corollary 3.2. Let $\mathcal{V}$ be a finitely generated variety. Then $\mathcal{V}$ is an atom in $\mathbf{L}(\mathcal{R} \mathcal{L})$ iff $\mathcal{V}=\mathbf{V}(\mathbf{L})$, for some finite strictly simple residuated lattice $\mathbf{L}$.

Proof. Let $\mathcal{V}$ be a minimal variety generated by a finite algebra $\mathbf{K}$. If $\mathbf{K}$ is not strictly simple, then there is a minimal non-trivial subalgebra $\mathbf{L}$ of $\mathbf{K}$. Since $\mathcal{V}$ is an atom, it is generated by $\mathbf{L}$. The converse is a direct consequence of the previous proposition; the necessary term exists because $\mathbf{L}$ is strictly simple and finite.

\section{Commutative atoms}

A generalized Boolean algebra is a lattice such that every principal filter is the lattice reduct of a Boolean algebra. Given a generalized Boolean algebra $\langle L, \wedge, \vee\rangle$, we denote by $e$ its top element and by $x / y=y \backslash x$ the complement of $y$ in the Boolean algebra $[x \wedge y, e]$; as usual, we set $[a, b]=\{c \in L \mid a \leq c \leq b\}$.

Lemma 4.1. If $\langle L, \vee, \wedge\rangle$ is a generalized Boolean algebra and $e$ is its top element, then the algebra $\langle L, \wedge, \vee, \wedge, \backslash, /, e\rangle$ is a residuated lattice. 
Proof. It is clear that $\langle L, \wedge, \vee\rangle$ is a lattice and $\langle L, \wedge, e\rangle$ is a monoid. Assume that $x \wedge y \leq z$ and let $a^{\prime}$ denote the complement in $[x \wedge y \wedge z, e]$ of an element $a \in[x \wedge y \wedge z, e]$. Then, $z^{\prime} \leq(x \wedge y)^{\prime}$, so $z^{\prime} \leq x^{\prime} \vee y^{\prime}$. Hence,

$$
z^{\prime} \wedge y \leq\left(x^{\prime} \vee y^{\prime}\right) \wedge y=\left(x^{\prime} \wedge y\right) \vee\left(y^{\prime} \wedge y\right)=x^{\prime} \wedge y \leq x^{\prime},
$$

consequently,

$$
x=x^{\prime \prime} \leq\left(z^{\prime} \wedge y\right)^{\prime}=z \vee y^{\prime}=\left(z \vee y^{\prime}\right) \wedge\left(y \vee y^{\prime}\right)=(z \wedge y) \vee y^{\prime} .
$$

Since $y^{\prime}$ is the complement of $y$ in $[x \wedge y \wedge z, e],(z \wedge y) \vee y^{\prime}$ is the complement of $y$ in $[(z \wedge y) \vee(x \wedge y \wedge z), e]=[z \wedge y, e]$, i.e., $(z \wedge y) \vee y^{\prime}=z / y$. Thus, $x \leq z / y$. Conversely, assume that $x \leq z / y$, where $z / y$ is the complement of $y$ in $[z \wedge y, e]$. Then, $x \wedge y \leq z / y \wedge y=z \wedge y \leq z$.

For the left division operation, we have $x \wedge y \leq z$ iff $y \wedge x \leq z$ iff $y \leq z / x=x \backslash z$, for all $x, y, z \in L$.

We call the residuated lattice $\langle L, \wedge, \vee, \wedge, \backslash, /, e\rangle$ a generalized Boolean algebra, as well, since the two algebras are definitionally equivalent. It is in this sense that we will use the term in the sequel. Thus, a generalized Boolean algebra is a residuated lattice such that every principal lattice filter is the lattice reduct of a Boolean algebra and multiplication coincides with meet. Note that other choices for multiplication can yield residuated lattices, as well, so we insist on the last condition. We denote the class of generalized Boolean algebras by $\mathcal{G B} \mathcal{A}$.

The only, up to isomorphism, two-element residuated lattice, which we denote by $\mathbf{2}$, is obviously a generalized Boolean algebra. The following proposition shows that the class $\mathcal{G B} \mathcal{A}$ is a finitely axiomatized variety and that it is generated by $\mathbf{2}$. Additionally, we provide a number of equational bases for it.

Proposition 4.2. Let $\mathbf{L}$ be a residuated lattice. The following statements are equivalent.

(1) $\mathbf{L}$ is a generalized Boolean algebra.

(2) $\mathbf{L}$ is in the variety $\mathbf{V}(\mathbf{2})$.

(3) L satisfies the identities

(a) $x y \approx x \wedge y$, and

(b) $x /(x \vee y) \vee(x \vee y) \approx e$.

(4) $\mathbf{L}$ satisfies the identities

(a) $x y \approx x \wedge y$, and

(b) $(x \wedge y) / y \vee y \approx e$.

(5) L satisfies the identities

(a) $x y \approx x \wedge y$, and

(b) $(y / x) \backslash y \approx x \vee y$. 
(6) L satisfies $x /(x \backslash y) \approx x \approx(y / x) \backslash x$.

Proof. We will show that $(1) \Rightarrow(3) \Rightarrow(4) \Rightarrow(2) \Rightarrow(5) \Rightarrow(1)$ and $(6) \Leftrightarrow(1)$.

$(1) \Rightarrow(3)$ : It follows directly from the definition of a generalized Boolean algebra.

$(3) \Rightarrow(4)$ : The identity (4b) follows from the identity (3b), by substituting $x \wedge y$ for $x$.

$(4) \Rightarrow(2)$ : Assume that $\mathbf{L}$ satisfies (4). We will show that it is a subdirect product of copies of $\mathbf{2}$. Let $P$ be a prime filter of $\mathbf{L}$ and let $f_{P}: L \rightarrow 2$ be defined by $f(x)=e$ iff $x \in P$. We will show that $f$ is a residuated lattice homomorphism. It is clear that $f_{P}$ is a lattice homomorphism, thus a monoid homomorphism as well. To prove that it preserves the division operations, given their behavior on $\mathbf{2}$, we only need to show that

$$
x / y \notin P \text { iff } x \notin P \text { and } y \in P .
$$

First observe that (4a) implies that $x \approx x \wedge e$. Assume that $x / y \notin P$ and $y \notin P$. Since $x / y=(x \wedge y) / y$, by Lemma 2.1(2) and (9), and $P$ is prime, we have $e=(x \wedge y) / y \vee y \notin P$, a contradiction. Assume that $x / y \notin P$ and $x \in P$. Since $x \leq x / y$ and $P$ is a filter, we have $x / y \in P$, a contradiction. Conversely, if $x \notin P$, $y \in P$ and $x / y \in P$, then $x \geq(x / y) \wedge y \in P$, by Lemma 2.1(4); hence $x \in P$, a contradiction.

Since $f_{P}$ is a homomorphism, $\operatorname{Ker}\left(f_{P}\right)$ is a congruence on $\mathbf{L}$. In order to prove that $\mathbf{L}$ is a subdirect product of copies of $\mathbf{2}$, we need only show that the intersection of the congruences above is the diagonal. This follows from the fact that in a distributive lattice any pair of elements $(a, b)$ can be separated by a prime filter; i.e., for all $a, b$, there exists a prime filter $P$ such that $a \in P$ and $b \notin P$, or $b \in P$ and $a \notin P$. Thus $\mathbf{L}$ is in $\mathbf{V}(\mathbf{2})$.

$(2) \Rightarrow(5)$ : It is trivial to check that 2 satisfies the identities in (5).

$(5) \Rightarrow(1)$ : Assume that $x, y$ are elements of $\mathbf{L}$, such that $x \leq y$. We will show that $x / y$ is the complement of $y$ in $[x, e]$. We have $x \leq x / y$, since $x \wedge y \leq x$; so $x \leq y \wedge(x / y)$. Moreover, $y \wedge(x / y) \leq x$, by Lemma 2.1(4), hence $y \wedge(x / y)=x$. Additionally,

$$
y \vee(x / y)=((x / y) / y) \backslash(x / y)=(x /(y \wedge y)) \backslash(x / y)=(x / y) \backslash(x / y)=e .
$$

It has already been mentioned that Brouwerian algebras have a distributive lattice reduct; so, $\mathbf{L}$ is distributive by (5a). Thus, every principal interval of $\mathbf{L}$ is a Boolean algebra.

$(1) \Leftrightarrow(6)$ : Having established the equivalence of (1) and (2), note that the algebra 2 satisfies the identity (6). Conversely, suppose the equation (6) holds in $\mathbf{L}$. For every element $y$ of $L$ we have $e=e /(e \backslash y)$, so $e \leq e / y$, i.e., $y \leq e$. $\mathrm{So}, \mathbf{L}$ is an integral residuated lattice. Moreover, for all $x, y \in L, x=x /(x \backslash y)$ 
implies $x \backslash(x \backslash y)=(x /(x \backslash y)) \backslash(x \backslash y)$. Thus, by (6) and Lemma 2.1(6), we have $x^{2} \backslash y=x \backslash y$. By setting $y=x^{2}$ and $y=x$ successively, we obtain $x^{2}=x$, for all $x \in L$. Together with integrality this gives $x y=x \wedge y$, for all $x, y \in L$, since

$$
x y \leq x e \wedge e y=x \wedge y=(x \wedge y)^{2} \leq x y .
$$

Thus, $\mathbf{L}$ is a Brouwerian algebra; hence, it has a distributive lattice reduct. Assume now that $y \leq x$. We will show that the complement of $x$ in $[y, e]$ is $x \backslash y$. Note that $y \leq x \backslash y$, by integrality, so $y \leq x \wedge x \backslash y$. On the other hand we have $x \wedge(x \backslash y) \leq y$, by Lemma 2.1(4), thus $x \wedge x \backslash y=y$. Moreover, using Lemma 2.1(3) and (2), we have

$$
\begin{aligned}
x \vee x \backslash y & =(x /(x \vee x \backslash y)) \backslash(x \vee x \backslash y) \\
& =(x / x \wedge x /(x \backslash y)) \backslash(x \vee x \backslash y) \\
& =(x / x \wedge x) \backslash(x \vee x \backslash y) \\
& \geq x \backslash x \geq e .
\end{aligned}
$$

So, $x \vee x \backslash y=e$.

Using (5) of the previous proposition, it is not difficult to show that $\mathcal{G B A}=$ $\mathcal{B} r \cap \mathcal{G M V}$. Moreover, by Corollary 3.2 and Proposition 4.2 the variety $\mathcal{G B A}$ is an atom. It is easy to see that it is the only atom below $\mathcal{B} r$.

Every finite strictly simple residuated lattice different than $\mathbf{2}$ has to have a top element different than $e$; otherwise $\{\perp, e\}$, where $\perp$ is the least element of the lattice, defines a subalgebra isomorphic to $\mathbf{2}$. Below we give an infinite list of examples of finite, commutative, totally ordered residuated lattices that are strictly simple and, consequently, generate distinct atoms in $\mathbf{L}(\mathcal{R L})$.

For every natural number $n$, set $T_{n}=\{\top, e\} \cup\left\{u_{k} \mid k \in \mathbb{N}_{n}^{+}\right\}$, where $\mathbb{N}_{n}^{+}=$ $\{1,2, \ldots, n\}$. Define an order relation on $T_{n}$, by $u_{k} \leq u_{l}$ iff $k \geq l$, and $u_{k}<e<\top$, for all positive natural numbers $k, l \leq n$; see Figure 1. Also, define multiplication by $x \top=\top x=x$, for all $x \neq e ; u_{k} u_{l}=u_{\min \{n, k+l\}}$, for all $k, l \in \mathbb{N}_{n}^{+}$; and two division operations by $x / y=\bigvee\left\{z \in T_{n} \mid z y \leq x\right\}$ and $y \backslash x=\bigvee\left\{z \in T_{n} \mid y z \leq x\right\}$. It is easy to verify that $\mathbf{T}_{n}=\left\langle T_{n}, \wedge, \vee, \cdot, \backslash, /, e\right\rangle$ is a residuated lattice. We set $u=u_{1}$. Note that $u_{k}=u^{k}$ for all $k \leq n$.

Lemma 4.3. The variety $\mathbf{V}\left(\mathbf{T}_{n}\right)$ is an atom in the subvariety lattice of $\mathcal{R} \mathcal{L}$, for every natural number $n$.

Proof. Note that the residuated lattice $\mathbf{T}_{n}$ is generated by each of its non-identity elements. If $x<e$, then $e / x=\top$; moreover, $e / \top=u_{1}$ and $u_{k}=u_{1}^{k}$, for all $k \leq n$. So, $\mathbf{T}_{n}$ is strictly simple; hence it generates an atom by Corollary 3.2. 


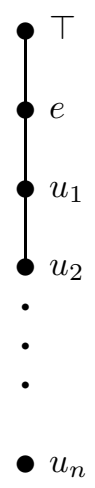

Figure 1. The residuated lattice $\mathbf{T}_{n}$.

We define the terms $\bar{\top}(x)=x \vee e / x$ and $\bar{u}(x)=e / \bar{\top}(x)$. Note that if $a \in$ $T_{n}-\{e\}$, then $\bar{\top}(a)=\top$ and $\bar{u}(a)=u$.

For every non-zero natural number $n$, we denote by $\mathbf{n}$ the integral residuated lattice defined by the monoid on the set $\left\{e, a, a^{2}, \ldots, a^{n-1}\right\}$, under the linear order $a^{n-1}<\cdots<a<e$. It is easy to see that $\mathbf{n}$ is a GMV-algebra.

Proposition 4.4. For every positive natural number $n$, the following list of equations is an equational basis for $\mathbf{V}\left(\mathbf{T}_{n}\right)$, relative to $\mathcal{R} \mathcal{L}$.

(1) $\lambda_{z}(x /(x \vee y)) \vee \rho_{w}(y /(x \vee y)) \approx e$

(2) $x^{n+1} \approx x^{n}$

(3) $(x \vee e)^{2} \approx(x \vee e)$

(4) $e /((x \vee e) \backslash e) \approx x \vee e$

(5) $(\bar{u}(x))^{n} \cdot x \approx(\bar{u}(x))^{n}$

(6) $x \wedge y \wedge e \approx((y \wedge e) /(x \wedge e) \wedge e)(x \wedge e)$

(7) $(x \wedge e)^{n} \approx(x \wedge e)^{n} /\left((x \wedge e)^{n} \backslash(y \wedge e)^{n} \wedge e\right) \wedge e$

(8) $\left[(x \wedge e)^{n} /(y \wedge e)^{n} \wedge e\right]^{2} \approx(x \wedge e)^{n} /(y \wedge e)^{n} \wedge e$

(9) $x y \approx y x$

(10) $(\bar{u}(x))^{n} /(\bar{u}(x))^{n-1} \wedge e \approx \bar{u}(x)$

Proof. It is easy to check that $\mathbf{V}\left(\mathbf{T}_{n}\right)$ satisfies all the equations. Conversely, let $\mathbf{L}$ be a subdirectly irreducible residuated lattice that satisfies the equations. $\mathbf{L}$ has to be a chain, because of the first equation and Theorem 2.3. Consider first the negative cone $\mathbf{L}^{-}$of $\mathbf{L}$. We will show that it is isomorphic to $\mathbf{m}$, for some $m \leq n$.

By (2), $\mathbf{L}^{-}$is $n$-potent. Equations (6) and (9) imply that $\mathbf{L}^{-}$is an integral commutative GBL-algebra; see $[2,10]$ for details. Note that the negative idempotent elements are of the form $x^{n}$ and that they form a subalgebra of $\mathbf{L}^{-}$. Indeed, they 
are closed under division by (8), obviously closed under the lattice operations, and the product of any two such elements is their meet - if $a \leq b$, then $a=a^{2} \leq a b \leq a$. By the identity (7) and Proposition 4.2(6), this subalgebra is a generalized Boolean algebra. Since it is also totally ordered it is isomorphic to $\mathbf{2}$.

We will show now that $\mathbf{L}^{-}$is generated by a single element as a monoid. Assume that there are non-identity elements $a \leq b$ that are not powers of a common element. Define $a_{1}=a, b_{1}=b, a_{k+1}=b_{k} \wedge b_{k} \backslash_{\mathbf{L}^{-}} a_{k}$ and $b_{k+1}=b_{k} \vee b_{k} \backslash_{\mathbf{L}^{-}} a_{k}$. Note that for every positive integer $k, a_{k} \leq b_{k} ; a_{k}=a_{k+1} b_{k+1}$, because of (9) and (6); and $b_{k}$ is an increasing sequence. So, $a=a_{1}=a_{2} b_{2}=a_{3} b_{3} b_{2}=\cdots=a_{n+1} b_{n+1} b_{n} b_{n-1} \cdots b_{3} b_{2} \leq$ $e\left(b_{n+1}\right)^{n}=\left(b_{n+1}\right)^{n}$. Since there are only two idempotent elements in $\mathbf{L}$, either $\left(b_{n+1}\right)^{n}=0$, or $\left(b_{n+1}\right)^{n}=e$. In the first case, $a=0=b^{n}$, so both $a$ and $b$ are powers of $b$. In the second case $b_{n+1}=e$. Since $b_{n+1}=b_{n} \vee b_{n} \backslash_{\mathbf{L}^{-}} a_{n}=b_{n-1} \vee$ $b_{n-1} \backslash_{\mathbf{L}^{-}} a_{n-1} \vee b_{n} \backslash_{\mathbf{L}^{-}} a_{n}=\cdots=b \vee b_{1} \backslash_{\mathbf{L}^{-}} a_{1} \vee \ldots \vee b_{n} \backslash_{\mathbf{L}^{-}} a_{n}$, we have $b_{l} \backslash_{\mathbf{L}^{-}} a_{l}=e$, for some $l$. Thus, $b_{l} \leq a_{l}$, so $a_{l}=b_{l}$. Using the fact that $b_{k} \in\left\{a_{k+1}, b_{k+1}\right\}$ and $a_{k}=a_{k+1} b_{k+1}$, for all $k$, and induction, it is not hard to see that both $b$ and $a$ are powers of $b^{l}$. So, $L^{-}=\left\{e, u, u^{2}, \ldots, u^{m}=\perp\right\}$, for some $m \leq n$.

Observe that $\mathbf{L}$ has a strictly positive element $a$. Otherwise, $\mathbf{L}$ would be integral, so $e / x=e$, for all $x \in L$, hence $\bar{\top}(x)=e$, i.e. $\bar{u}(x)=e$. In that case, (5) would imply $e \cdot x=e$, for all $x \in A$, a contradiction. By (3), we get $a^{2}=a$. For every strictly positive element $b$ of $L$, we have $u=e u \leq b u$. If $e \leq b u$, we have $e \leq$ beu $\leq$ bbuu $\leq \cdots \leq b^{m} u^{m}=b^{m} \perp=\perp$, a contradiction. So $b u=u$, hence $b \backslash e=u$. Using equation (4), we have $b=e /(b \backslash e)=e / u$, so there is a unique strictly positive element $T=e / u$ in $\mathbf{L}$. Finally, note that if $m<n$, then $u^{n}=u^{n-1}$, thus $u^{n} / u^{n-1} \wedge e=e$, a fact that contradicts (10). Thus, $\mathbf{L}$ is isomorphic to $\mathbf{T}_{n}$.

Corollary 4.5. There are infinitely many commutative, representable atoms in the subvariety lattice of $\mathcal{R} \mathcal{L}$.

The cardinality of the class of all representable, commutative atoms in $\mathbf{L}(\mathcal{R} \mathcal{L})$ remains unknown to us. We conjecture, though, that there are only countably many such atoms.

Working toward a partial description of finite, commutative, strictly simple, residuated chains, we note that they have properties similar to the ones of the algebras $\mathbf{T}_{n}$.

Proposition 4.6. Let $\mathbf{L}$ be a finite, commutative, representable, strictly simple residuated lattice and let $\top$ be its top element. Then $x \top=x$, for all $x \neq e$. Moreover, $\top$ covers e and e covers $e / T$, if $\top \neq e$.

Proof. Obviously, $\mathbf{L}$ is a subdirectly irreducible element of $\mathcal{R} \mathcal{L}^{C}$, so $\mathbf{L}$ is chain. If $\mathbf{L} \cong \mathbf{2}$, then the conclusion is obvious. Otherwise, $\mathbf{L}$ has a top element $T \neq e$. 
If $e=e / T$, then $e \leq e / T$, i.e., $T \leq e$, a contradiction. So, $e \neq e / T$. Note that $e / T=e / T^{2}=(e / T) / T$, by Lemma 2.1(6), so $e / T \leq(e / T) / T$; hence $(e / \top) \top \leq e / \top$. On the other hand, $e / \top \leq(e / \top) \top$, since $e \leq \top$; so $(e / \top) \top=$ $(e / T)$.

It is easy to show that if $x \top=x$ and $y \top=y$, then $x y \top=x y,(x / y) \top=x / y$ and $(e / x) \top=e / x$. By the assumption of strict simplicity, for every element of $a \neq e$ of $\mathbf{L}$, there exists a term $t_{a}$, such that $a=t_{a}(\top)$. By induction on the complexity of $t_{a}$, it can be shown that $x \top=x$, for all $x \neq e$.

To show that $e$ is covered by $T$, note that if $x>e$, then $\top \leq \top x=x$. It is obvious that $e / \top \leq e$. If $x<e$, then $\top x \leq e$, so $x \leq e / \top$, hence $e$ is a cover of $e / T$.

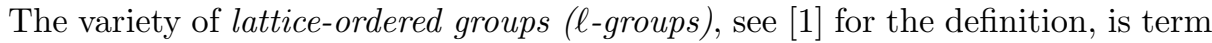
equivalent to the subvariety $\mathcal{L G}$ of residuated lattices axiomatized by $(e / x) x \approx e$, see $[2,10]$ for details. It is well known and easy to observe that the variety $\mathbf{V}(\mathbb{Z})$ generated by the $\ell$-group of the integers under addition is the only $\ell$-group atom. It is shown in [2] that $\mathbf{V}\left(\mathbb{Z}^{-}\right)$, the variety generated by the negative cone of $\mathbb{Z}$, is the only atom below the variety of negative cones of $\ell$-groups. Both of these atoms are cancellative. Below we note that they actually are the only atoms below the variety of cancellative residuated lattices.

The following proposition shows that $\mathbf{V}\left(\mathbb{Z}^{-}\right)$and $\mathcal{L G}$ form a splitting pair in the subvariety lattice of cancellative residuated lattices; i.e., for every cancellative variety $\mathcal{V}$ of residuated lattices, either $\mathcal{V} \subseteq \mathcal{L G}$ or $\mathbf{V}\left(\mathbb{Z}^{-}\right) \subseteq \mathcal{V}$.

Proposition 4.7. For every cancellative residuated lattice, either it has $\mathbb{Z}^{-}$as a subalgebra or it is an $\ell$-group.

Proof. Let $\mathbf{L}$ be a cancellative residuated lattice. Since division is order reversing in the denominator, for every negative element $a, e \leq e / a$. Hence, either there exists a strictly negative element $a$ of $L$ such that $e / a=e$, or for every strictly negative element $x$ of $L, e<e / x$. It is easy to see that in the first case the subalgebra generated by $a$ is isomorphic to $\mathbb{Z}^{-}$. Since $a<e$, we get $a^{n+1} \leq a^{n}$, for every natural number $n$. Actually, $a^{n+1}<a^{n}$, because otherwise, we would get $a=e$, by cancellativity. Moreover, $a^{k+m} / a^{m}=a^{k}$ and $a^{m} / a^{m+k}=e$, for all natural numbers $m, k$. Thus, the set of all powers of $a$ defines a subalgebra of $\mathbf{L}$ isomorphic to $\mathbb{Z}^{-}$.

In the second case for every element $a$ of $L$, consider the element $x=(e / a) a$; we have $x \leq e$, by Lemma 2.1(4). It cannot be strictly negative because $e / x=$ $e /(e / a) a=(e / a) /(e / a)=e$, by Lemma 2.1(6) and cancellativity; so $x=e$. Thus, $\mathbf{L}$ is an $\ell$-group. 
The following corollary answers an open question (Problem 8.5) of [12].

Corollary 4.8. The varieties $\mathbf{V}(\mathbb{Z})$ and $\mathbf{V}\left(\mathbb{Z}^{-}\right)$are the only cancellative atoms in the subvariety lattice of $\mathcal{R} \mathcal{L}$.

\section{Idempotent atoms}

In view of Corollary 4.8, it makes sense to investigate the other end of the spectrum of atoms, i.e., varieties that are $n$-potent for some $n$. We will provide a continuum of idempotent, representable atoms.

For every set of integers $S$, set $N_{S}=\left\{a_{i} \mid i \in \mathbb{Z}\right\} \cup\left\{b_{i} \mid i \in \mathbb{Z}\right\} \cup\{e\}$. We define an order relation on $N_{S}$ by $b_{i}<b_{j}<e<a_{k}<a_{l}$, for all $i, j, k, l \in \mathbb{Z}$ such that $i<j$ and $k>l$; see Figure 2. Obviously, this is a total order on $N_{S}$. We also define a multiplication operation by

$$
a_{i} a_{j}=a_{\min \{i, j\}}, \quad b_{i} b_{j}=b_{\min \{i, j\}}
$$

and

$$
b_{j} a_{i}=\left\{\begin{array}{ll}
b_{j} & \text { if } j<i \text { or } i=j \in S \\
a_{i} & \text { if } j>i \text { or } i=j \notin S
\end{array}, a_{i} b_{j}= \begin{cases}a_{i} & \text { if } i<j \text { or } i=j \in S \\
b_{j} & \text { if } i>j \text { or } i=j \notin S\end{cases}\right.
$$

Finally, we define two division operations on $N_{S}$, by $x / y=\bigvee\{z \mid z y \leq x\}$ and $y \backslash x=\bigvee\{z \mid y z \leq x\}$; note that the joins exist.

It is easy to see that multiplication is associative and residuated by the division operations. So, we can define a residuated lattice $\mathbf{N}_{S}$ with underlying set $N_{S}$ and operations the ones described above.

We will investigate for which sets $S$ the variety generated by $\mathbf{N}_{S}$ is an atom in the subvariety lattice of residuated lattices.

We define the following residuated lattice terms:

$$
\begin{gathered}
\ell(x)=x \backslash e, r(x)=e / x, \\
t(x)=e / x \vee x \backslash e, \\
m(x)=\ell \ell(x) \wedge \ell r(x) \wedge r \ell(x) \wedge r r(x), \\
p(x)=\ell \ell(x) \vee \ell r(x) \vee r \ell(x) \vee r r(x) .
\end{gathered}
$$

Moreover, we consider three binary relations defined by,

$$
\begin{aligned}
& x \stackrel{r}{\rightarrow} y \Leftrightarrow r(x)=y, \\
& x \underset{\ell}{\rightarrow} y \Leftrightarrow \ell(x)=y,
\end{aligned}
$$




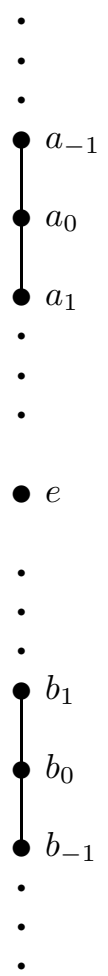

Figure 2. The residuated lattice $\mathbf{N}_{S}$, where $S \subseteq \mathbb{Z}$.

$$
x \rightarrow y \Leftrightarrow r(x)=y \text { or } \ell(x)=y .
$$

A word over $\{0,1\}$ is a function $w: A \rightarrow\{0,1\}$, where $A$ is a subinterval of $\mathbb{Z}$; A is called the support, $\operatorname{supp}(w)$, of $w$. We call $w$ finite (infinite, bi-infinite) if $|A|<\omega\left(A=\mathbb{N}^{+}, A=\mathbb{Z}\right.$, respectively). If $w$ is a word and $v$ a finite word, we say $v$ is a subword of $w$, if there exists an integer $k$, such that $v(i)=w(i+k)$ for all $i \in \operatorname{supp}(v)$. Note that the characteristic function $w_{S}$ of a subset $S$ of $\mathbb{Z}$ is a bi-infinite word. For two words $w_{1}, w_{2}$, define $w_{1} \leq w_{2}$ iff every finite subword of $w_{1}$ is a subword of $w_{2}$. Obviously, $\leq$ is a pre-order. Define $w_{1} \cong w_{2}$ iff $w_{1} \leq w_{2} \leq w_{1}$. We call a bi-infinite word $w$ minimal with respect to the pre-order $\leq$, if, for every bi-infinite word $w^{\prime}, w^{\prime} \leq w$ implies $w \cong w^{\prime}$. 
In the following we write $x \prec y$ for the fact that $x$ is covered by $y$; i.e., $x<y$ and for every $z$, if $x \leq z \leq y$, then $z=x$ or $z=y$.

Lemma 5.1. The following properties hold for $\mathbf{N}_{S}$, for every $S \subseteq \mathbb{Z}$.

(1) For all $i \in \mathbb{Z}, m\left(b_{i}\right)=b_{i-1}, p\left(b_{i}\right)=b_{i+1}, m\left(a_{i}\right)=a_{i+1}, p\left(a_{i}\right)=a_{i-1}$. Moreover, $t\left(a_{i}\right)=b_{i}$ and $t\left(b_{i}\right)=a_{i}$.

(2) For all $x, y, x \leq y$ or $y \leq x$.

(3) For every $x,\{x t(x), t(x) x\}=\{x, t(x)\}$.

(4) If $x<e<y$, then $m(x) \prec x \prec p(x)<e<m(y) \prec y \prec p(y)$ and $t(y)<e<$ $t(x)$.

(5) For every $x, m(t(x))=t(p(x)), p(t(x))=t(m(x)), m(p(x))=p(m(x))=x$ and $t(t(x))=x$.

(6) If $x$ is negative, then $x y=y x= \begin{cases}x & \text { for } x \leq y<t(x) \\ y & \text { for } y \leq x \text { or } t(x)<y .\end{cases}$

If $x$ is positive, then $x y=y x= \begin{cases}x & \text { for } t(x)<y \leq x \\ y & \text { for } y<t(x) \text { or } x \leq y .\end{cases}$

(7) For all $x, y ; x \wedge y, x \vee y, x y \in\{x, y\}$.

(8) For all $x, y ; x / y, y \backslash x \in\{x, m(x), p(x), t(x), m(t(x)), t(y), m(t(y)), p(t(y)\}$.

(9) For every finite word $v$ there exists a universal first order formula $\phi_{v}$, such that $v$ is not a subword of $w_{S}$ iff $\phi_{v}$ is satisfied in $\mathbf{N}_{S}$.

Proof. It is easy to see that

$$
\begin{array}{ll}
b_{i-1} \overleftarrow{\ell} a_{i} \underset{\ell}{\stackrel{r}{\longrightarrow}} b_{i} \stackrel{r}{\rightarrow} a_{i+1} & (i \in S) \\
b_{i-1} \stackrel{r}{\leftarrow} a_{i} \stackrel{r}{\stackrel{r}{\longrightarrow}} b_{i} \underset{\ell}{\longrightarrow} a_{i+1} & (i \notin S)
\end{array}
$$

It follows directly that $t\left(b_{i}\right)=a_{i} \vee a_{i+1}=a_{i}$ and $t\left(a_{i}\right)=b_{i-1} \vee b_{i}=b_{i}$. Moreover,

$$
\left\{r\left(r\left(b_{i}\right)\right), r\left(\ell\left(b_{i}\right)\right), \ell\left(r\left(b_{i}\right)\right), \ell\left(\ell\left(b_{i}\right)\right)\right\}=\left\{b_{i-1}, b_{i}, b_{i+1}\right\},
$$

so $m\left(b_{i}\right)=b_{i-1}$ and $p\left(b_{i}\right)=b_{i+1}$. Similarly, $m\left(a_{i}\right)=a_{i+1}$ and $p\left(a_{i}\right)=a_{i-1}$; so, (1) holds. Moreover, (2) is obvious from the definition; (3)-(7) follow from (1); and (8) is routine to check. Finally for (9), the first order formula associated to a finite word $v$ is

$$
\begin{gathered}
\phi_{v}=\left(\forall x_{1}, \ldots, x_{n}, y_{1}, \ldots, y_{n}\right)\left\{\left[\left(x_{1} \prec x_{2} \prec \cdots \prec x_{n}<e<y_{n} \prec \cdots \prec y_{1}\right)\right.\right. \\
\left.\left.\&\left(t\left(x_{1}\right) \approx y_{1} \& \cdots \& t\left(x_{n}\right) \approx y_{n}\right)\right] \rightarrow \neg\left(x_{1} y_{1} \approx s_{1} \& \cdots \& x_{n} y_{n} \approx s_{n}\right)\right\},
\end{gathered}
$$

where $n$ is the length of $v ; s_{i}=x_{i}$, if $v(i)=1$; and $s_{i}=y_{i}$, if $v(i)=0$. Note that $\phi_{v}$ is equivalent to a universally quantified formula in the language of residuated lattices. 
Corollary 5.2. The residuated lattice $\mathbf{N}_{S}$ is strictly simple, for every set of integers $S$.

Proof. For all $a, b \in N_{S}-\{e\},(a, b)$ is in the transitive closure of the relation $\rightarrow$ defined above. Thus, $\mathbf{N}_{S}$ is strictly simple.

Lemma 5.3. Every non-trivial, one-generated subalgebra of an ultrapower of $\mathbf{N}_{S}$ is isomorphic to $\mathbf{N}_{S^{\prime}}$, for some set of integers $S^{\prime}$.

Proof. Every first order formula true in $\mathbf{N}_{S}$ is also true in an ultrapower of it. Since properties (2)-(8) of Lemma 5.1 can be expressed as first order formulas, they hold in every ultrapower of $\mathbf{N}_{S}$.

By property (2), any ultrapower $\mathbf{B}$ of $\mathbf{N}_{S}$ is totally ordered, so the same holds for every subalgebra of $\mathbf{B}$. Let $\mathbf{A}$ be a non-trivial one-generated subalgebra of $\mathbf{B}$ and let $a$ be a generator for $\mathbf{A}$. The element $a$ can be taken to be negative, since if $a$ is positive, $t(a)$ is negative, by property (4), and it generates $\mathbf{A}$, because, by property (5), we have $t(t(a))=a$.

By properties (7) and (8), $A$ is the set of evaluations of the terms composed by the terms $m, p, t$ and the constant term $e$. By property (5), these compositions reduce to one of the forms $m^{n}(x), p^{n}(x), p^{n}(t(x))$ and $m^{n}(t(x))$, for $n$ a natural number.

Set $b_{-n}=m^{n}(a), b_{n}=p^{n}(a), a_{-n}=p^{n}(t(a))$ and $a_{n}=m^{n}(t(a))$, for all natural numbers $n$. By the remark above, $A$ consists of exactly these elements together with $e$. Define a subset $S^{\prime}$ of $\mathbb{Z}$, by $m \in S^{\prime}$ iff $b_{m} a_{m}=b_{m}$ and consider the map $f: A \rightarrow$ $N_{S^{\prime}}=\left\{b_{i}^{\prime} \mid i \in \mathbb{Z}\right\} \cup\left\{a_{i}^{\prime} \mid i \in \mathbb{Z}\right\} \cup\left\{e^{\prime}\right\}$, defined by $f\left(b_{i}\right)=b_{i}^{\prime}, f\left(a_{i}\right)=a_{i}^{\prime}, f(e)=e^{\prime}$. By property (4), $f$ is an order isomorphism and, consequently, a lattice isomorphism. Moreover, it is easy to check that $f$ is a monoid homomorphism, using properties (3) and (6). Every lattice isomorphism preserves existing joins, so $f$ preserves the two division operations. Thus, $\mathbf{A}$ is isomorphic to $\mathbf{N}_{S^{\prime}}$.

Theorem 5.4. Let $\mathbf{A}$ be a one-generated residuated lattice and $S$ a subset of $\mathbb{Z}$. Then, $\mathbf{A} \in \mathbf{H S P}_{u}\left(\mathbf{N}_{S}\right)$ iff $\mathbf{A} \cong \mathbf{N}_{S^{\prime}}$, for some $S^{\prime}$ such that $w_{S^{\prime}} \leq w_{S}$.

Proof. Let $S^{\prime}$ be a set of integers, such that $w_{S^{\prime}} \leq w_{S}$. Also, let $\mathbf{B}=\left(\mathbf{N}_{S}\right)^{\mathbb{N}} / \mathcal{F}$, where $\mathcal{F}$ is an ultrafilter over $\mathbb{N}$ that extends the filter of co-finite subsets, and $N_{S}=\left\{b_{i} \mid i \in \mathbb{Z}\right\} \cup\left\{a_{i} \mid i \in \mathbb{Z}\right\} \cup\{e\}$. We will show that $\mathbf{N}_{S^{\prime}} \in \mathbf{I S P}_{u}\left(\mathbf{N}_{S}\right)$.

For every natural number $n$, define the finite approximations $v_{n}$ of the bi-infinite word $w_{S^{\prime}}$, by $v_{n}(i)=w_{S^{\prime}}(i)$, for all $i \in[-n, n]_{\mathbb{Z}}$. Since, $w_{S^{\prime}} \leq w_{S}$, the words $v_{n}$ are subwords of $w_{S}$, so for every natural number $n$ there exists an integer $K_{n}$, such that $v_{n}(i)=w_{S}\left(K_{n}+i\right)$, for all $i \in \operatorname{supp}\left(v_{n}\right)=[-n, n]_{\mathbb{Z}}$.

Let $\bar{b}=\left(b_{K_{n}}\right)_{n \in \mathbb{N}}$, where $b_{K_{n}} \in N_{S}$. By Lemma 5.3 , the subalgebra of $\mathbf{B}$ generated by $\tilde{b}=[\bar{b}]$, the equivalence class of $\bar{b}$ under $\mathcal{F}$, is isomorphic to $\mathbf{N}_{\tilde{S}}, N_{\tilde{S}}=$ $\left\{\tilde{b}_{i} \mid i \in \mathbb{Z}\right\} \cup\left\{\tilde{a}_{i} \mid i \in \mathbb{Z}\right\} \cup\{\tilde{e}\}$, for some subset $\tilde{S}$ of $\mathbb{Z}$. We identify the subalgebra 
generated by $\tilde{b}$ with $\mathbf{N}_{\tilde{S}}$ and, without loss of generality, we choose $\tilde{S}$ such that $\tilde{b}_{0}=\tilde{b}$. We will show that $\tilde{S}=S^{\prime}$.

We pick representatives $\bar{b}_{m}$ and $\bar{a}_{m}$, for $\tilde{b}_{m}$ and $\tilde{a}_{m}$, respectively, for all $m \in \mathbb{Z}$, and we adopt a double subscript notation for their coordinates. So, there exist $\bar{b}_{m n}$ and $\bar{a}_{m n}$ in $\mathbf{N}_{S}$, such that $\tilde{b}_{m}=\left[\bar{b}_{m}\right]=\left[\left(\bar{b}_{m n}\right)_{n \in \mathbb{N}}\right]$ and $\tilde{a}_{m}=\left[\bar{a}_{m}\right]=\left[\left(\bar{a}_{m n}\right)_{n \in \mathbb{N}}\right]$.

It is easy to prove that $\tilde{b}_{m}=\left[\left(b_{K_{n}+m}\right)_{n \in \mathbb{N}}\right]$ and $\tilde{a}_{m}=\left[\left(a_{K_{n}+m}\right)_{n \in \mathbb{N}}\right]$, using the definition of $\tilde{b}$, Lemma 5.1(1), basic induction and the following facts:

$$
\begin{gathered}
\tilde{a}_{m}=t\left(\tilde{b}_{m}\right)=t\left(\left[\left(\bar{b}_{m n}\right)_{n \in \mathbb{N}}\right]\right)=\left[\left(t\left(\bar{b}_{m n}\right)\right)_{n \in \mathbb{N}}\right] \\
\tilde{b}_{m+1}=p\left(\tilde{b}_{m}\right)=p\left(\left[\left(\bar{b}_{m n}\right)_{n \in \mathbb{N}}\right]\right)=\left[\left(p\left(\bar{b}_{m n}\right)\right)_{n \in \mathbb{N}}\right] \\
\tilde{b}_{m-1}=m\left(\tilde{b}_{m}\right)=m\left(\left[\left(\bar{b}_{m n}\right)_{n \in \mathbb{N}}\right]\right)=\left[\left(m\left(\bar{b}_{m n}\right)\right)_{n \in \mathbb{N}}\right]
\end{gathered}
$$

Now, for $|m|<n$, i.e., $m \in \operatorname{supp}\left(v_{n}\right)$, we have

$$
\begin{aligned}
K_{n}+m \in S & \Leftrightarrow w_{S}\left(K_{n}+m\right)=1 \\
& \Leftrightarrow v_{n}(m)=1 \\
& \Leftrightarrow w_{S^{\prime}}(m)=1 \\
& \Leftrightarrow m \in S^{\prime} .
\end{aligned}
$$

Since, $b_{K_{n}+m} a_{K_{n}+m}=b_{K_{n}+m}$ exactly when $K_{n}+m \in S$, we get that if $|m|<n$, then $b_{K_{n}+m} a_{K_{n}+m}=b_{K_{n}+m}$ is equivalent to $m \in S^{\prime}$.

In other words,

$$
\{n|| m \mid<n\} \subseteq\left\{n \mid b_{K_{n}+m} a_{K_{n}+m}=b_{K_{n}+m} \Leftrightarrow m \in S^{\prime}\right\} .
$$

Since the first set is in $\mathcal{F}$, so is the second one. It is not hard to check that this means that: $\left\{n \mid b_{K_{n}+m} a_{K_{n}+m}=b_{K_{n}+m}\right\} \in \mathcal{F}$ is equivalent to $m \in S^{\prime}$. So, $\tilde{b}_{m} \tilde{a}_{m}=\tilde{b}_{m}$ is equivalent to $m \in S^{\prime}$; hence $m \in \tilde{S}$ iff $m \in S^{\prime}$. Thus, $\tilde{S}=S^{\prime}$

For the converse, we will prove the implication for $\mathbf{A} \in \mathbf{S P}_{u}\left(\mathbf{N}_{S}\right)$. This is sufficient since under a homomorphism every one generated subalgebra will either map isomorphically or to the identity element, because of the strictly simple nature of the algebras $\mathbf{N}_{S^{\prime}}$. Let $\mathbf{A}$ be a subalgebra of an ultrapower of $\mathbf{N}_{S}$. By Lemma 5.3, $\mathbf{A}$ is isomorphic to $\mathbf{N}_{S^{\prime}}$, for some subset $S^{\prime}$ of $\mathbb{Z}$.

To show that $w_{S^{\prime}} \leq w_{S}$ it suffices to show that, for every finite word $v$, if $v$ is not a subword of $w_{S}$, then it is not a subword of $w_{S^{\prime}}$ either. If $v$ is not a subword of $w_{S}$, then $\mathbf{N}_{S}$ satisfies $\phi_{v}$ of Lemma 5.1(9); hence so does every ultrapower of $\mathbf{N}_{S}$. Since $\phi_{v}$ is universally quantified it is also satisfied by any subalgebra of an ultrapower of $\mathbf{N}_{S}$ and in particular by $\mathbf{N}_{S^{\prime}}$. Thus, $v$ is not a subword of $w_{S^{\prime}}$.

Corollary 5.5. Let $S, S^{\prime}$ be sets of integers, then

(1) $\mathbf{V}\left(\mathbf{N}_{S^{\prime}}\right) \subseteq \mathbf{V}\left(\mathbf{N}_{S}\right)$ if and only if $w_{S^{\prime}} \leq w_{S}$, and

(2) if $w_{S}$ is minimal with respect to $\leq$, then $\mathcal{V}=\mathbf{V}\left(\mathbf{N}_{S}\right)$ is an atom in the subvariety lattice of $\mathcal{R} \mathcal{L}$. 
Proof. 1) If $w_{S^{\prime}} \leq w_{S}$ then, by Theorem 5.4, $\mathbf{N}_{S^{\prime}} \in \mathbf{H S P}_{u}\left(\mathbf{N}_{S}\right) \subseteq \mathbf{V}\left(\mathbf{N}_{S}\right)$, so $\mathbf{V}\left(\mathbf{N}_{S^{\prime}}\right) \subseteq \mathbf{V}\left(\mathbf{N}_{S}\right)$. Conversely, if $\mathbf{V}\left(\mathbf{N}_{S^{\prime}}\right) \subseteq \mathbf{V}\left(\mathbf{N}_{S}\right)$, then $\mathbf{N}_{S^{\prime}} \in \mathbf{V}\left(\mathbf{N}_{S}\right)$. $\mathbf{N}_{S^{\prime}}$ is subdirectly irreducible, by Lemma 5.2, so, by Jónsson's Lemma, $\mathbf{N}_{S^{\prime}} \in \mathbf{H S P}_{u}\left(\mathbf{N}_{S}\right)$. By Theorem 5.4, $w_{S^{\prime}} \leq w_{S}$.

2) If $\mathbf{L}$ is a subdirectly irreducible of $\mathcal{V}$, then $\mathbf{L} \in \mathbf{H S P}_{u}\left(\mathbf{N}_{S}\right)$, by Jónsson's Lemma. Every one-generated subalgebra $\mathbf{A}$ of $\mathbf{L}$ is a member of $\mathbf{S H S P}_{u}\left(\mathbf{N}_{S}\right) \subseteq$ $\mathbf{H S P}_{u}\left(\mathbf{N}_{S}\right)$, because $\mathbf{S H} \leq \mathbf{H S}$; so, by Theorem 5.4, $\mathbf{A}$ is isomorphic to some $\mathbf{N}_{S^{\prime}}$, where $w_{S^{\prime}} \leq w_{S}$. Since $w_{S}$ is minimal with respect to the pre-order $\leq$, we have $w_{S^{\prime}} \cong w_{S}$; hence $\mathbf{V}\left(\mathbf{N}_{S^{\prime}}\right)=\mathbf{V}\left(\mathbf{N}_{S}\right)$, by (1). Thus, $\mathcal{V}=\mathbf{V}\left(\mathbf{N}_{S^{\prime}}\right)=\mathbf{V}(\mathbf{A}) \subseteq \mathbf{V}(\mathbf{L}) \subseteq$ $\mathcal{V}$. Since $\mathcal{V}=\mathbf{V}(\mathbf{L})$, for every subdirectly irreducible $\mathbf{L}$ in $\mathcal{V}, \mathcal{V}$ is an atom.

The following corollary answers Problem 8.6 of [12].

Corollary 5.6. There are uncountably many atoms in the subvariety lattice of $\mathcal{R} \mathcal{L}^{C} \cap \operatorname{Mod}\left(x^{2} \approx x\right)$.

Proof. In [14] one can find a study on infinite and bi-infinite words. Among other things, words that are minimal with respect to the $\leq$ preorder are constructed. Such words have been re-discovered in different areas of mathematics and have numerous applications.

One way to construct such a word is to consider a line $\ell$ on the plane. The lower mechanical word $w_{\ell}$ corresponding to the line $\ell$ is obtained by approximating the line from below by a broken line, see Figure 3 . The admissible line segments of the approximating broken line have to have endpoints $(x, y)$ and $\left(x+1, y+k_{x}\right)$, such that $x, y$ are integers and $k_{x}=0$ or $k_{x}=1$. The word $w_{\ell}$ is defined by $w_{\ell}(x)=k_{x}$, for all $x \in \mathbb{Z}$. It is shown in [14] that if $\ell$ has irrational slope then $w_{\ell}$ is minimal. Moreover, if we consider only lines that contain the origin and have irrational slope, we obtain a class of size continuum such that all words are minimal and pairwise incomparable.

For a more precise definition of the lower mechanical word associated with a line and for the proofs of the facts mentioned above, the reader is referred to [14].

The proof of the previous result relies heavily on the fact that the generating algebras are not commutative. If we add the restriction of commutativity, or even the weaker condition $e / x \approx x \backslash e$, we get only finitely many atoms, actually only two, even without the assumption that they are representable.

Theorem 5.7. The varieties $\mathbf{V}(2)$ and $\mathbf{V}\left(\mathbf{T}_{1}\right)$ are the only atoms below the variety $\operatorname{Mod}\left(x^{2} \approx x, e / x \approx x \backslash e\right)$.

Proof. Assume $\mathbf{A}$ is a non trivial member of $\operatorname{Mod}\left(x^{2} \approx x, e / x \approx x \backslash e\right)$ and let $a$ be a negative element of $\mathbf{A}$. Since $a \leq e$, we have $e \leq e / a$. If $e / a=e$, then $\{e, a\}$ is a subuniverse of $\mathbf{A}$. If $e<e / a$, set $T=e / a$ and $b=e / T$. We will show 


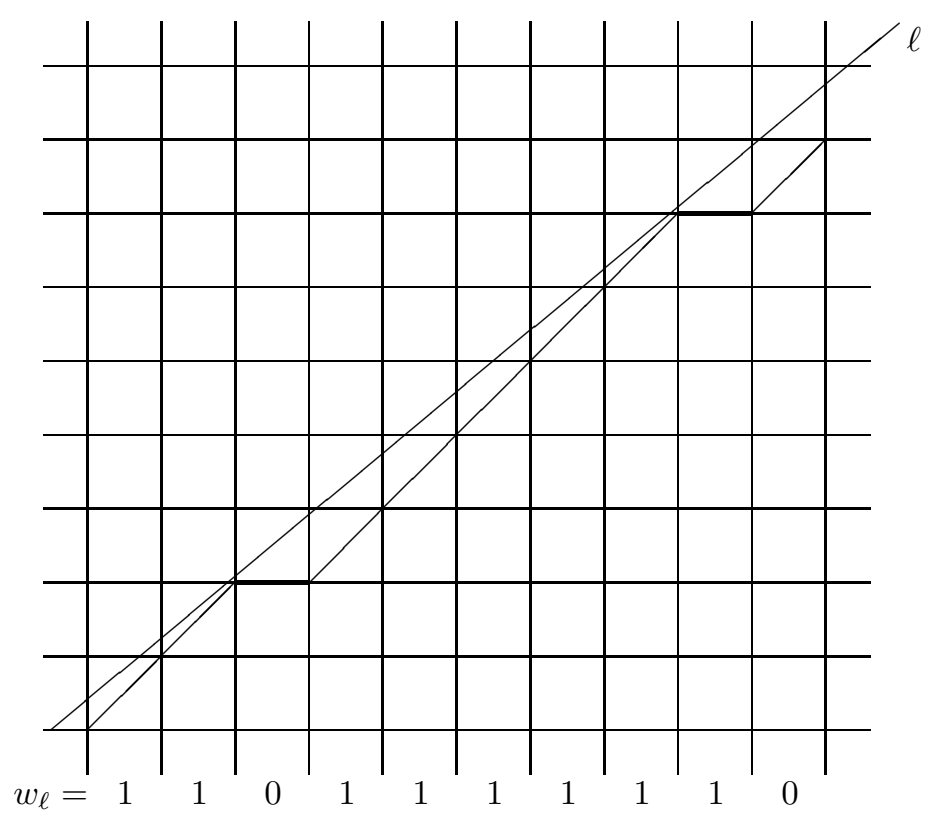

Figure 3 . The word $w_{\ell}$ corresponding to a line $\ell$.

that $\{b, e, T\}$ is a subuniverse of $\mathbf{A}$. Note that $a T=a(e / a)=a(a \backslash e) \leq e$. So, $a \leq e / T=b$. We have $b \leq b T=(e / T) T \leq e$ and $b T=b b T \leq b$, so $b T=b$. Since $e / T=T \backslash e$, we also get $T b=b$. Additionally, $T \leq e / b$. Also, $(e / b) a \leq(e / b) b \leq e$, so $e / b \leq e / a=T$; thus, $T=e / b$. Moreover, $b \leq b / T \leq(b / T) T \leq b$, so $b / T=b$. Finally, $a \leq a a \leq b a \leq a$, so $T / b=(e / a) / b=e / b a=e / a=T$.

We conclude this section with a remark on varieties generated by algebras similar to the ones of the form $\mathbf{N}_{S}$.

For every infinite word $w$, set $N_{w}=\left\{a_{i} \mid i \in \mathbb{N}^{+}\right\} \cup\left\{b_{i} \mid i \in \mathbb{N}\right\} \cup\{e\}$, where $\mathbb{N}^{+}$ denotes the set of positive integers. We define an order on $N_{w}$, by $b_{i}<b_{j}<e<$ $a_{k}<a_{l}$, for all $i, j \in \mathbb{N}$ and $k, l \in \mathbb{N}^{+}$, such that $i<j$ and $k>l$; see Figure 4 . Obviously, this is a total order on $N_{w}$. Let $S_{w}=\left\{n \in \mathbb{N}^{+} \mid w(n)=1\right\}$ and define multiplication on $N_{w}$, by

$$
a_{i} a_{j}=a_{\min \{i, j\}}, \quad b_{i} b_{j}=b_{\min \{i, j\}}
$$

and

$$
b_{j} a_{i}=\left\{\begin{array}{ll}
b_{j} & \text { if } j<i \text { or } i=j \in S_{w} \\
a_{i} & \text { if } j>i \text { or } i=j \notin S_{w}
\end{array}, a_{i} b_{j}= \begin{cases}a_{i} & \text { if } i<j \text { or } i=j \in S_{w} \\
b_{j} & \text { if } i>j \text { or } i=j \notin S_{w}\end{cases}\right.
$$


Finally, we define two division operations on $N_{S}$, by $x / y=\bigvee\{z \mid z y \leq x\}$ and $y \backslash x=\bigvee\{z \mid y z \leq x\}$; note that the joins exist.

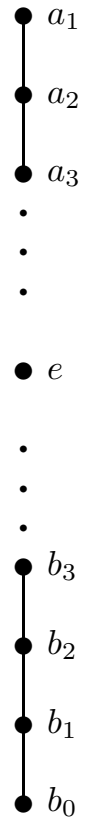

Figure 4 . The residuated lattice $\mathbf{N}_{w}$, where $w$ is infinite.

It is easy to see that multiplication is associative and residuated by the division operations. So, we can define a residuated lattice $\mathbf{N}_{w}$ with underlying set $N_{w}$ and operations the ones described above.

For uniformity, if $w$ is a bi-infinite word and $S_{w}=\{n \in \mathbb{Z} \mid w(n)=1\}$, we set $\mathbf{N}_{w}=\mathbf{N}_{S_{w}}$. We prove the following result that complements Corollary 5.5(1).

\section{Corollary 5.8.}

(1) Assume that $w^{\prime}$ is a bi-infinite word and $w$ an infinite word. Then,

(a) $\mathbf{V}\left(\mathbf{N}_{w^{\prime}}\right) \subseteq \mathbf{V}\left(\mathbf{N}_{w}\right)$ if and only if $w^{\prime} \leq w$; and

(b) if $w \cong w^{\prime}$, then $\mathbf{V}\left(\mathbf{N}_{w^{\prime}}\right)$ is covered by $\mathbf{V}\left(\mathbf{N}_{w}\right)$.

(2) If $w^{\prime}$ and $w$ are infinite words then $\mathbf{V}\left(\mathbf{N}_{w^{\prime}}\right)$ and $\mathbf{V}\left(\mathbf{N}_{w}\right)$ are distinct incomparable varieties. 
Sketch of proof. (1)(a) First note that $\mathbf{N}_{w}$ is strictly simple and, consequently, subdirectly irreducible. Following the ideas in the proof of Lemma 5.3, we can show that the one-generated subalgebras of an ultrapower of $\mathbf{N}_{w}$, where $w$ is an infinite word, are either isomorphic to $\mathbf{N}_{w}$ or of the form $\mathbf{N}_{S}$, for $S \subseteq \mathbb{Z}$. Moreover, we can show that $w_{S} \leq w$, using the formula of Lemma 5.1(9). Homomorphic images preserve these facts, because $\mathbf{N}_{w}$ and $\mathbf{N}_{S}$ are strictly simple. Conversely, if $w_{S} \leq w$, then we can show that $\mathbf{N}_{S}$ is isomorphic to a subalgebra of the ultrapower $\left(\mathbf{N}_{w}\right)^{\mathbb{N}}$, mimicking the proof of Theorem 5.4.

(b) It is clear from the above that the only subdirectly irreducible algebras in $\mathbf{V}\left(\mathbf{N}_{w}\right)$ are $\mathbf{N}_{w}$ and $\mathbf{N}_{S}$, for $w_{S} \leq w$. Moreover, we know that the only subdirectly irreducible algebras in $\mathbf{V}\left(\mathbf{N}_{w^{\prime}}\right)$ are isomorphic to $\mathbf{N}_{S}$, for $w_{S} \leq w^{\prime}$, i.e. $w_{S} \leq w$. Thus, $\mathbf{V}\left(\mathbf{N}_{w^{\prime}}\right)$ is covered by $\mathbf{V}\left(\mathbf{N}_{w}\right)$.

(2) This follows from the analysis on the subdirectly irreducibles mentioned in the previous paragraph.

\section{Residuated bounded-lattices}

A residuated bounded-lattice is an algebra $\mathbf{L}=\langle L, \wedge, \vee, \cdot, \backslash, /, e, \perp\rangle$ such that $\langle L, \wedge, \vee, \cdot, \backslash, /, e\rangle$ is a residuated lattice and $\perp$ is its least element. By Lemma 2.1 every residuated bounded-lattice has a top element $T=\perp / \perp$. Thus, the $\perp$-free reduct of a residuated bounded-lattice is a bounded residuated lattice. We denote the variety of residuated bounded-lattices by $b \mathcal{R} \mathcal{L}$. Residuated bounded-lattices have been considered in the context of logic, see $[15,16]$, because of the natural interpretation of the bounds as absolute truth and falsehood.

Note that the congruence lattice of a residuated bounded-lattice is isomorphic to the congruence lattice of its $\perp$-free reduct, because the expansion of the language by constants does not affect the congruence generation. Therefore, the congruences of a residuated bounded-lattice correspond to the convex normal subalgebras of its $\perp$-free reduct.

In what follows we will identify specific bounded residuated lattices with their residuated bounded-lattice expansions. For example, we will denote by $\mathbf{2}$ the only, up to isomorphism, two-element residuated bounded-lattice.

Note that Proposition 3.1 and Corollary 3.2 extend to the bounded case, as well. So, $\mathbf{V}(\mathbf{2})$ and $\mathbf{V}\left(\mathbf{T}_{n}\right)$, where $n$ is a positive natural number, are atoms of $\mathbf{L}(b \mathcal{R} \mathcal{L})$.

Below, we comment on the third level of $\mathbf{L}(b \mathcal{R} \mathcal{L})$, whose elements we call almost minimal (non-trivial) varieties, following [13]. In particular, we explain why minimal varieties of $\mathbf{L}(\mathcal{R} \mathcal{L})$ give rise to almost minimal varieties of $\mathbf{L}(b \mathcal{R} \mathcal{L})$ that are covers of atoms like $\mathbf{V}(\mathbf{2})$ or $\mathbf{V}\left(\mathbf{T}_{1}\right)$. 
Let $\mathbf{L}$ be a residuated lattice and $\perp, \top \notin L$. We define the set $\mathbf{b}(L)=L \cup\{\perp, \top\}$ and extend the operations of $\mathbf{L}$ to $\mathbf{b}(L)$ as follows. For all $a \in L, b \in L \cup\{\top\}$ and $c \in \mathbf{b}(L)$, the operations $\wedge, \vee$ are given by $\perp<a<\top$; multiplication is defined by $b \top=\top b=\top, \perp c=c \perp=\perp$; and the division operations by $\top / b=b \backslash \top=$ $c / \perp=\perp \backslash c=\top, a / \top=\top \backslash a=\perp / b=b \backslash \perp=\perp$. The above operations define the algebra $\mathbf{b}(\mathbf{L})=\langle\mathbf{b}(L), \wedge, \vee, \cdot, \backslash, /, e, \perp\rangle$.

If $\mathbf{L}$ is an integral residuated lattice, we can modify the above construction and obtain an algebra $\mathbf{d}(\mathbf{L})$, where $\mathbf{d}(L)=L \cup\{\perp\}$ and the extension of the operations is defined in a similar way.

The constructions obtained using the operators $\mathbf{b}$ and $\mathbf{d}$ produce residuated bounded-lattices or bounded residuated lattices (depending on whether we include $\perp$ in the type), as it is shown in [9], and are special cases of a more general construction that we describe below. In particular, $\mathbf{d}$ preserves integrality.

We call an element $a$ in an algebra $\mathbf{A}$ irreducible with respect to an $n$-ary operation $f$ of $\mathbf{A}$, if, for all $a_{1}, a_{2}, \ldots, a_{n} \in A, f\left(a_{1}, a_{2}, \ldots, a_{n}\right)=a$ implies $a_{i}=a$, for some $i$. We call a totally irreducible, if it is irreducible with respect to all the operations of $\mathbf{A}$.

Let $\mathbf{K}$ and $\mathbf{L}$ be residuated lattices and assume that $\mathbf{K}$ is integral or the identity element of $\mathbf{K}$ is totally irreducible; also, assume that either $\mathbf{L}$ is integral, or there is no element $k \in K$ such that $e / k=e$ or $k \backslash e=e$. In this case we say that $\mathbf{K}$ is admissible by $\mathbf{L}$. A class $\mathcal{K}$ is admissible by a class $\mathcal{L}$, if every algebra of $\mathcal{K}$ is admissible by every algebra of $\mathcal{L}$.

Let $K[L]=\left(K-\left\{e_{\mathbf{K}}\right\}\right) \cup L$, and extend the operations of $\mathbf{K}$ and $\mathbf{L}$ to $K[L]$ by $l \star k=e_{\mathbf{K}} \star_{\mathbf{K}} k$ and $k \star l=k \star_{\mathbf{K}} e_{\mathbf{K}}$, for $k \in\left(K-\left\{e_{\mathbf{K}}\right\}\right), l \in L$ and $\star \in\{\wedge, \vee, \cdot, \backslash, /\}$. We will show that $\mathbf{K}[\mathbf{L}]=\left\langle K[L], \wedge, \vee, \cdot, \backslash, /, e_{\mathbf{L}}\right\rangle$ is a residuated lattice. We extend the operator $\mathbf{K}$ on classes of algebras that admit $\mathbf{K}$ and use the same notation for the case where $\mathbf{K}$ is a residuated bounded-lattice; in this case we view $\mathbf{K}[\mathbf{L}]$ as a residuated bounded-lattice. Note that for every residuated lattice $\mathbf{L}, \mathbf{b}(\mathbf{L})=\mathbf{T}_{1}[\mathbf{L}]$; if $\mathbf{L}$ is integral, then $\mathbf{d}(\mathbf{L})=\mathbf{2}[\mathbf{L}]$.

The residuated lattices $\mathbf{T}_{n}$, for $n$ a positive natural number, and $\mathbf{N}_{w}$, for $w$ an infinite or bi-infinite word are examples of algebras that are admissible by all residuated lattices.

Lemma 6.1. Assume that $\mathbf{K}$ is a residuated bounded-lattice, $\mathbf{L}$ is a residuated lattice and that $\mathbf{K}$ is admissible by $\mathbf{L}$. Then,

(1) the algebra $\mathbf{K}[\mathbf{L}]$ is a residuated bounded-lattice; 
(2) the congruence lattice of $\mathbf{K}[\mathbf{L}]$ is isomorphic to the coalesced ordinal sum of the congruence lattice of $\mathbf{L}$ and the congruence lattice of $\mathbf{K}$. Thus, $\mathbf{K}[\mathbf{L}]$ is subdirectly irreducible iff $\mathbf{L}$ is subdirectly irreducible, or $\mathbf{L} \cong \mathbf{1}$ and $\mathbf{K}$ is subdirectly irreducible.

Proof. (1) The proof for $\mathbf{K}=\mathbf{T}_{1}$ and $\mathbf{K}=\mathbf{2}$ can be found in [9]. The proof for an arbitrary admissible $\mathbf{K}$ is straight-forward.

(2) By Theorem 2.2, the congruence lattice of $\mathbf{K}[\mathbf{L}]$ is isomorphic to the lattice of convex normal subalgebras of its $\perp$-free reduct. Note that $L$ is normal in $\mathbf{K}[\mathbf{L}]$, because for all $a \in L$ and $k \in K-\{e\}, \rho_{k}(a)=k a / k \wedge e=k / k \wedge e=e$ and similarly $\lambda_{k}(a)=e$. Actually, $\mathbf{L}$ is a convex normal subalgebra of the $\perp$-free reduct of $\mathbf{K}[\mathbf{L}]$. Assume that $\mathbf{M}$ is a convex (normal) subalgebra of the $\perp$-free reduct of $\mathbf{K}[\mathbf{L}]$, such that $M \nsubseteq L L$. It is not hard to see that, without loss of generality, we can chose $k \in M-L$, such that $k<e$. Thus, $e \leq e / k$. If $e<e / k$, then $k, e / k \in K$, and $k<$ $e<e / k$. So, $k<l<e / k$, for all $l \in L$. Consequently, $L \subseteq M$, since $k, e / k \in M$. If $e / k=e$, then $\mathbf{L}$ is integral, since $\mathbf{K}$ is admissible by $\mathbf{L}$, and $k \in K$. Thus, $k<l \leq e$, for all $l \in L$. So, $L \subseteq M$, since $k \in M$. Consequently, for every convex (normal) subalgebra $\mathbf{M}$ of $\mathbf{K}[\mathbf{L}]$, either $L \subseteq M$ or $M \subseteq L$. Additionally, it can be verified that if $\mathbf{M}$ is a convex normal subalgebra of the $\perp$-free reduct of $\mathbf{K}[\mathbf{L}]$ such that $L \subseteq M$, then $(M-L) \cup\{e\}$, defines a convex normal subalgebra of $\mathbf{K}$. Conversely, for every convex normal subalgebra $\mathbf{N}$ of $\mathbf{K},(N-\{e\}) \cup L$ defines a convex normal subalgebra of the $\perp$-free reduct of $\mathbf{K}[\mathbf{L}]$. Moreover, these correspondences are mutually inverse lattice isomorphisms. Consequently, the lattice of convex normal subalgebras of the $\perp$-free reduct of $\mathbf{K}[\mathbf{L}]$ is isomorphic to the coalesced ordinal sum of the lattices of convex normal subalgebras of $\mathbf{L}$ and $\mathbf{K}$. Statement (2), then, follows directly from Theorem 2.2.

Lemma 6.2. Assume that $\mathcal{L}$ is a class of residuated lattices and that $\mathbf{K}$ is a finite strictly simple residuated bounded-lattice admissible by $\mathcal{L}$. Then,

(1) $\mathbf{O}(\mathbf{K}[\mathcal{L}])=\mathbf{K}[\mathbf{O}(\mathcal{L})]$, where $\mathbf{O}$ is any of the operators $\mathbf{I P}_{u}, \mathbf{S}$ or $\mathbf{H}$.

(2) $(\mathbf{V}(\mathbf{K}[\mathcal{L}]))_{S I}=\mathbf{K}\left[(\mathbf{V}(\mathcal{L}))_{S I}\right] \cup \mathbf{I}(\mathbf{K})$.

Proof. (1) Assume first that $\mathbf{O}=\mathbf{I P}_{u}$. If $\mathbf{L}_{i} \in \mathcal{L}, i \in I$ and $\mathcal{F}$ is an ultrafilter over $I$, then $\left(\prod_{i \in I} \mathbf{K}\left[\mathbf{L}_{i}\right]\right) / \mathcal{F} \cong \mathbf{K}\left[\left(\prod_{i \in I} \mathbf{L}_{i}\right) / \mathcal{F}\right]$. This is true, because every element of the the left hand side has a representative ( $I$-sequence) that has all or none of its terms in $K-\{e\}$. Thus we can separate the elements in ones of type $\mathbf{K}$ and in ones of type $\mathcal{L}$. The elements of type $\mathcal{L}$ form a subalgebra of the left hand side isomorphic to $\left(\prod_{i \in I} \mathbf{L}_{i}\right) / \mathcal{F}$. On the other hand, every element of type $\mathbf{K}$ is of the form $\left[(k)_{i \in I}\right]_{\mathcal{F}}$, for $k \in K-\{e\}$, since $\mathbf{K}$ is finite. Thus, the left hand side is isomorphic to $\mathbf{K}\left[\left(\prod_{i \in I} \mathbf{L}_{i}\right) / \mathcal{F}\right]$.

For the case $\mathbf{O}=\mathbf{S}$, note that every subalgebra (computed in $b \mathcal{R} \mathcal{L}$ ) of $\mathbf{K}[\mathbf{L}]$, for $\mathbf{L} \in \mathcal{L}$, is equal to $\mathbf{K}$ of a subalgebra (computed in $\mathcal{R} \mathcal{L}$ ) of $\mathbf{L}$, by the very 
construction of $\mathbf{K}[\mathbf{L}]$ and the fact that $\mathbf{K}$ is strictly simple. Conversely, for every subalgebra $\mathbf{L}^{\prime}$ (computed in $\mathcal{R} \mathcal{L}$ ) of $\mathbf{L}, \mathbf{K}\left[\mathbf{L}^{\prime}\right]$ is a subalgebra (computed in $b \mathcal{R} \mathcal{L}$ ) of $\mathbf{K}[\mathbf{L}]$.

Finally, assume that $\mathbf{O}=\mathbf{H}$. Note that no non-constant homomorphism on $\mathbf{K}[\mathbf{L}]$ collapses any pair of elements of $\mathrm{K}$, because in that case it would also collapse the identity element with the bottom element and the image of the map would be trivial. Moreover, if a homomorphism $h$ collapses an element of $\mathbf{K}$ with an element of $\mathbf{L}$, then $h$ is constant. To see this assume that $h(a)=h(k)$, for some $a \in L$ and $k \in K-\{e\}$. Then, $h(a \vee k)=h(a)=h(a \wedge k)$ and, by the construction of $\mathbf{K}[\mathbf{L}]$, $a \wedge k \in K-\{e\}$ or $a \vee k \in K-\{e\}$. Thus, we may assume that $h(a)=h(k)$, for some $a \in L$ and $k \in K-\{e\}$ such that $k<a$ or $a<k$. In the first case $k<e$ and

$$
h(k)=h(k / a \wedge e)=h(k) / h(a) \wedge e=h(a) / h(a) \wedge e=e .
$$

In the second case $e<k, e / k<e$ and

$$
h(e / k)=h(a / k \wedge e)=h(a) / h(k) \wedge e=h(a) / h(a) \wedge e=e .
$$

Since $\mathbf{K}$ lacks non-trivial subalgebras, for every element $k^{\prime} \in K-\{e\}$ there is a term $\overline{k^{\prime}}(x)$ such that $\overline{k^{\prime}}(x)=k^{\prime}$, for $x \neq e$. Thus, for every $k^{\prime} \in K-\{e\}, h\left(k^{\prime}\right)=e$, i.e. the image of $h$ is trivial. Consequently, the image of $\mathbf{K}[\mathbf{L}]$ under $h$ is of the form $\mathbf{K}[\mathbf{M}]$, where $\mathbf{M}=h(\mathbf{L})$. Thus, the homomorphic image of $\mathbf{K}[\mathbf{L}]$ is in $\mathbf{K}[\mathbf{H}(\mathcal{L})]$. Conversely, if $\mathbf{M}$ is a homomorphic image (computed in $\mathcal{R} \mathcal{L}$ ) of an algebra $\mathbf{L}$ of $\mathcal{L}$, then $\mathbf{K}[\mathbf{M}]$ is a homomorphic image (computed in $b \mathcal{R} \mathcal{L}$ ) of $\mathbf{K}[\mathbf{L}]$.

(2) By Jónsson's Lemma for congruence distributive varieties and (1), we have $(\mathbf{V}(\mathbf{K}[\mathcal{L}]))_{S I} \subseteq \mathbf{H S P}_{u}(\mathbf{K}[\mathcal{L}])=\mathbf{K}\left[\mathbf{H S P}_{u}(\mathcal{L})\right]$. Assume that $\mathbf{L} \in(\mathbf{V}(\mathbf{K}[\mathcal{L}]))_{S I}$. Then $\mathbf{L}=\mathbf{K}[\mathbf{M}]$, for some $\mathbf{M} \in \mathbf{H S P}_{u}(\mathcal{L}) \subseteq \mathbf{V}(\mathcal{L})$. Moreover, since $\mathbf{K}[\mathbf{M}]$ is subdirectly irreducible, we have that $\mathbf{M}$ is subdirectly irreducible or $\mathbf{M} \cong \mathbf{1}$, by Lemma 6.1(3). Consequently, $\left.\mathbf{L} \in \mathbf{K}\left[(\mathbf{V}(\mathcal{L}))_{S I}\right] \cup \mathbf{I}(\mathbf{K}[\mathbf{1}])\right)=\mathbf{K}\left[(\mathbf{V}(\mathcal{L}))_{S I}\right] \cup$ $\mathbf{I}(\mathbf{K})$. Conversely, if $\mathbf{L} \in \mathbf{K}\left[(\mathbf{V}(\mathcal{L}))_{S I}\right] \cup \mathbf{I}(\mathbf{K})$, then $\mathbf{L}$ is subdirectly irreducible by Lemma 6.1(3). Note that $\mathbf{K}\left[(\mathbf{V}(\mathcal{L}))_{S I}\right] \cup \mathbf{I}(\mathbf{K}) \subseteq \mathbf{K}\left[\mathbf{H S P}_{u}(\mathcal{L})\right]=\mathbf{H S P}_{u}(\mathbf{K}[\mathcal{L}]) \subseteq$ $\mathbf{V}(\mathbf{K}[\mathcal{L}])$. Thus, $\mathbf{L} \in(\mathbf{V}(\mathbf{K}[\mathcal{L}]))_{S I}$.

Note that $\mathbf{K}$ does not commute with the operator $\mathbf{P}$.

Theorem 6.3. If $\mathcal{V}$ is an atom in the subvariety lattice of $\mathcal{R} \mathcal{L}$ and $\mathbf{K}$ a finite, strictly simple residuated bounded-lattice admissible by $\mathcal{V}$, then $\mathbf{V}(\mathbf{K}[\mathcal{V}])$ is a cover of $\mathbf{V}(\mathbf{K})$ in $\mathbf{L}(b \mathcal{R} \mathcal{L})$. Moreover, this correspondence is injective.

Proof. Let $\mathcal{V}$ be an atom in in the subvariety lattice of $\mathcal{R} \mathcal{L}$. Using Lemma 6.2(2), we have

$$
(\mathbf{V}(\mathbf{K}[\mathcal{V}]))_{S I}=\mathbf{K}\left[(\mathbf{V}(\mathcal{V}))_{S I}\right] \cup \mathbf{I}(\mathbf{K})=\mathbf{K}\left[\mathcal{V}_{S I}\right] \cup \mathbf{I}(\mathbf{K})
$$

Thus, $\mathbf{V}(\mathbf{K}) \subseteq \mathbf{V}(\mathbf{K}[\mathcal{V}])$, and every subdirectly irreducible algebra in $\mathbf{V}(\mathbf{K}[\mathcal{V}])$ is either isomorphic to $\mathbf{K}$ or of the form $\mathbf{K}[\mathbf{L}]$, for some subdirectly irreducible $\mathbf{L}$ of $\mathcal{V}$. 
For every subdirectly irreducible $\mathbf{K}[\mathbf{L}]$ of $\mathbf{V}(\mathbf{K}[\mathcal{V}])$ not isomorphic to $\mathbf{K}$, we have

$$
(\mathbf{V}(\mathbf{K}[\mathcal{V}]))_{S I}=\mathbf{K}\left[\mathcal{V}_{S I}\right] \cup \mathbf{I}(\mathbf{K})=\mathbf{K}\left[(\mathbf{V}(\mathbf{L}))_{S I}\right] \cup \mathbf{I}(\mathbf{K})=(\mathbf{V}(\mathbf{K}[\mathbf{L}]))_{S I},
$$

by Lemma 6.2(2); hence $\mathbf{V}(\mathbf{K}[\mathcal{V}])=\mathbf{V}(\mathbf{K}[\mathbf{L}])$. Thus, $\mathbf{V}(\mathbf{K}[\mathcal{V}])$ is a cover of $\mathbf{V}(\mathbf{K})$.

Moreover, if $\mathcal{V}_{1} \cap \mathcal{V}_{2} \subseteq \mathbf{V}(\mathbf{K})$, then the varieties $\mathbf{V}\left(\mathbf{K}\left[\mathcal{V}_{1}\right]\right)$ and $\mathbf{V}\left(\mathbf{K}\left[\mathcal{V}_{2}\right]\right)$ cannot share a common subdirectly irreducible different than $\mathbf{K}$. Otherwise, if $\mathbf{K}[\mathbf{L}]$ is that subdirectly irreducible, then $\mathbf{L}$ is a common subdirectly irreducible of $\mathcal{V}_{1}$ and $\mathcal{V}_{2}$. In particular, if $\mathcal{V}_{1}$ and $\mathcal{V}_{2}$ are distinct atoms, then $\mathbf{V}\left(\mathbf{K}\left[\mathcal{V}_{1}\right]\right)$ and $\mathbf{V}\left(\mathbf{K}\left[\mathcal{V}_{2}\right]\right)$ are distinct covers of $\mathbf{V}(\mathbf{K})$.

Corollary 6.4. The varieties $\mathbf{V}\left(\mathbf{T}_{1}\left[\mathbf{N}_{S}\right]\right)$, where $w_{S}$ is minimal, form a class of continuum many idempotent, representable, almost minimal varieties of $\mathbf{L}(b \mathcal{R} \mathcal{L})$.

The existence of continuum many almost minimal varieties of $\mathbf{L}(b \mathcal{R} \mathcal{L})$ was already known. In [13] a continuum of idempotent, commutative, integral varieties and a continuum of representable, commutative, integral varieties that are almost minimal in $\mathbf{L}(b \mathcal{R} \mathcal{L})$ are constructed. However, none of the varieties constructed is both idempotent and representable.

As mentioned before, a continuum of atoms of $\mathbf{L}(\mathcal{R L})$ is given in [12]. The varieties are generated by totally ordered, bounded residuated lattices. The same algebras, viewed as residuated bounded-lattices, generate a continuum of representable atoms in $\mathbf{L}(b \mathcal{R} \mathcal{L})$ that satisfy $x^{4} \approx x^{3}$, but are not idempotent.

Below we give a continuum of idempotent, representable atoms in $\mathbf{L}(b \mathcal{R} \mathcal{L})$.

Corollary 6.5. The varieties $\mathbf{V}\left(\mathbf{N}_{w}\right)$, where $w$ is an infinite word, form a class of continuum many atoms in $\mathbf{L}(b \mathcal{R} \mathcal{L})$.

Proof. (Sketch) We work as in Corollary 5.5. Now, we focus on the zero-generated subalgebra of an ultrapower of $\mathbf{N}_{w}$, and show that it is isomorphic to $\mathbf{N}_{w}$. Moreover, it has to be contained in every subalgebra of the ultrapower. If a homomorphic image of a subalgebra of an ultrapower collapses any two elements of the isomorphic copy of $\mathbf{N}_{w}$, it has to collapse the whole subalgebra. To see that, first recall that $\mathbf{N}_{w}$ is strictly simple, so we may assume that $h\left(b_{0}\right)=h\left(b_{1}\right)$ under the homomorphism $h$. Then, $h\left(b_{0}\right)=h\left(b_{0} / b_{1} \wedge e\right)=h\left(b_{0}\right) / h\left(b_{1}\right) \wedge e=h\left(b_{1}\right) / h\left(b_{1}\right) \wedge e=e$. Since, the multiplicative identity is the least element only in a one-element residuated lattice, we have that the homomorphic image is trivial. The rest of the proof proceeds as in Corollary 5.5.

The author would like to thank Peter Jipsen and the anonymous referee for their helpful remarks and suggestions. 


\section{REFERENCES}

[1] M. Anderson and T. Feil, Lattice-Ordered Groups: an introduction, D. Reidel Publishing Company, 1988.

[2] P. Bahls, J. Cole, N. Galatos, P. Jipsen and C. Tsinakis, Cancellative Residuated Lattices, Algebra Universalis 50 (2003), 83-106.

[3] W. Blok and C. van Alten, On the finite embeddability property for residuated lattices, pocrims and BCK-algebras, Algebra \& substructural logics (Tatsunokuchi, 1999). Rep. Math. Logic No. 34, (2000), 159-165.

[4] K. Blount, On the structure of residuated lattices, Ph.D. Thesis, Dept. of Mathematics, Vanderbilt University, Nashville, TN, 1999.

[5] K. Blount and C. Tsinakis, The structure of Residuated Lattices, Internat. J. Algebra Comput. 13 (2003), 437-461.

[6] N. Galatos, The undecidability of the word problem for distributive residuated lattices, Ordered algebraic structures (J. Martinez, ed.), Kluwer Academic Publishers, Dordrecht, 2002, pp. 231-243.

[7] N. Galatos, Equational bases for joins of residuated-lattice varieties, Studia Logica 76 (2004), 227-240.

[8] N. Galatos, Varieties of residuated lattices, Ph.D. thesis, Dept. of Mathematics, Vanderbilt University, Nashville, TN, 2003.

[9] N. Galatos and J. Raftery, Adding Involution to Residuated Structures, Studia Logica, 77 (2004), 181-207.

[10] N. Galatos and C. Tsinakis, Generalized $M V$-algebras, to appear in Journal of Algebra.

[11] J. Hart, L. Rafter and C. Tsinakis, The Structure of Commutative Residuated Lattices, Internat. J. Algebra Comput. 12 (2002), 509-524.

[12] P. Jipsen and C. Tsinakis, A survey of Residuated Lattices, Ordered Algebraic Structures (J. Martinez, ed.), Kluwer Academic Publishers, Dordrecht, 2002, pp. 19-56.

[13] T. Kowalski and H. Ono, Residuated lattices: An algebraic glimpse at logics without contraction, JAIST Reports, 2000.

[14] M. Lothair, Algebraic combinatorics on words, Encyclopedia of Mathematics and its Applications, Cambridge University Press, 2002.

[15] H. Ono, Semantics for substructural logics, Substructural logics (Tübingen, 1990), Stud. Logic Comput., 2, Oxford Univ. Press, New York, 1993, pp. 259-291.

[16] H. Ono, Substructural Logics and Residuated Lattices - an introduction, Trends in Logic vol. 20, "50 Years of Studia Logic" (V.F. Hendricks and J. Malinowski eds.), Kluwer Academic Publishers, 2003, pp. 177-212.

[17] J. Raftery and C. van Alten, Rule Separation and Embedding Theorems for Logics Without Weakening, Studia Logica 76 (2004), 241-274.

[18] M. Ward and R. P. Dilworth, Residuated Lattices, Transactions of the AMS 45 (1939), $335-354$

Nikolaos Galatos

School of Information Science, Japan Advanced Institute of Science and Technology, 1-1

Asahidai, Tatsunokuchi, Ishikawa, 923-1292, Japan

e-mail: galatos@jaist.ac.jp 